Pathologe 2007 · 28:474-478

DOI 10.1007/s00292-007-0942-6

Online publiziert: 22 . September 2007

(c) Springer Medizin Verlag 2007

K. Glatz

Institut für Pathologie, Basel, Schweiz

\title{
Molekulare Heterogenität maligner Melanome
}

\section{Häufige genetische \\ Veränderungen}

Im Folgenden werden die häufigsten beim malignen Melanom beobachteten genetischen Veränderungen beschrieben (- Abb. 1).

\section{MAPK-Signalweg}

In verschiedenen Studien wurde gezeigt, dass der MAPK- (Mitogen-aktivierteProteinkinase-) Signalweg eine entscheidende Rolle bei der Melanomentstehung einnimmt. Durch onkogene Mutationen aktiviertes N-RAS aktiviert die Serin-Threonin-Proteinkinase Raf, welche in den 3 Isoformen A-Raf, B-Raf und C-Raf vorkommt. RAF phosphoryliert und aktiviert MEK, eine weitere Serin-Threonin-Proteinkinase. MEK schließlich phosphoryliert und aktiviert die Kinasen ERK1 und ERK2. Letztere sind in die Regulation der Zellproliferation involviert und tragen zur vermehrten Proliferation von Tumorzellen bei [27]. Die Aktivierung des MAPKSignalweges scheint eine zentrale Rolle bei der Entstehung melanozytärer Tumoren einzunehmen. Eine Aktivierung dieses Weges findet sich bei rund $80 \%$ aller Melanome, wobei entweder eine somatische Missense-Mutation von $B R A F$ oder seltener eine Mutation von $N-R A S$ vorliegt $[14,16]$. Onkogene BRAF-Mutationen finden sich aber nicht nur bei malignen Melanomen, sondern auch bei melanozytären Nävi $[14,39]$. Während gutartige Nävi auf die Aktivierung dieses Signalweges mit einer stabilen Wachstumsinhibition (Seneszenz) antworten [34], wachsen maligne Melanome weiter und akkumulieren weitere genetische Veränderungen.

$\mathrm{p} 16^{\text {INK4a }}$ und $\mathrm{p} 14^{\text {ARF }}$

Das Gen CDKN2A auf Chromosom 9p21 kodiert über einen alternativen Leserahmen 2 verschiedene Proteine, $\mathrm{p}_{1} 6^{\mathrm{INK} 4 \mathrm{a}}$ und p $14{ }^{\text {ARF }}$. Diese beiden Proteine haben Tumorsuppressorfunktion. Nach Phosphorylierung des Retinoblastomproteins $(\mathrm{Rb})$ durch die Cyklin-abhängigen Proteinkinasen $\mathrm{CDK}_{4}$ und CDK6 kann die Zelle in die S-Phase des Zellzyklus eintreten. $\mathrm{p}_{1} 6^{\mathrm{INK} 4 \mathrm{a}}$ hemmt die $\mathrm{CDK}_{4} / 6$ vermittelte Phosphorylierung von $\mathrm{Rb}$ und verhindert dadurch die Zellzyklusprogression. p14 ${ }^{\mathrm{ARF}}$ hemmt MDM 2, welches den Abbau von $\mathrm{p} 53$ beschleunigt und erhöht dadurch die Konzentration des Tumorsuppressors p53. Der Verlust von p16 $6^{\mathrm{INK} 4 \mathrm{a}}$ und p14 ${ }^{\mathrm{ARF}}$ prädisponiert zur Melanomentwicklung [9]. Keimbahnmutationen des $C D K N_{2} A$-Gens werden bei $25 \%$ aller Melanomfamilien beobachtet [41].

\section{Microphthalmia-assoziierter}

Transkriptionsfaktor (MITF)

MITF reguliert Entwicklung, Funktion und Überleben von Melanozyten, indem es verschiedene Differenzierungsgene und Gene der Zellzyklusprogression steuert [31]. In den meisten Zellen ist ein Zellzyklusarrest für die normale Differenzierung notwendig. MITF löst den Zellzyklusarrest durch die Aktivierung von $\mathrm{p} 16^{\mathrm{INK} 4 \mathrm{a}}$ und die Hypophosphorylierung des Rb-Proteins aus. Diese Verbindung von Zellzyklusarrest und Differenzierung erklärt möglicherweise, weshalb 
beim malignen Melanom häufig Alterationen von $\mathrm{p}_{1} 6^{\mathrm{INK} 4 \mathrm{a}}$ nachweisbar sind [32].

Amplifikationen des MITF-Gens wurden bei primären (10\%) und metastasierten (15\%) Melanomen nachgewiesen. In Melanomzellinien waren MITF-Amplifikationen assoziiert mit einer BRAF-Mutation und $\mathrm{p}_{1} 6^{\mathrm{INK} 4 \mathrm{a}}$-Inaktivierung [21]. Dies deutet möglicherweise darauf hin, dass die Amplifikation von MITF in Kombination mit einer Deregulation der Zellzykluskontrolle und der vermehrten Aktivierung des MAPK-Signalweges die Tumorbildung und/oder das Tumorüberleben fördert. Experimentell konnte gezeigt werden, dass reduzierte MITF-Aktivität die Empfindlichkeit von Melanomzellen für konventionelle Chemotherapeutika steigert [21].

\section{Phosphatidylinositol-3'- Kinase- (PI3 K-)Signalweg}

PTEN ist eine Lipid-Protein-Phosphatase mit den Eigenschaften eines Tumorsuppressorgens [50]. PTEN inhibiert die Phosphorylierung und damit Aktivierung der Serin-Threonin-Kinase Akt durch $\mathrm{PI}_{3} \mathrm{~K}$. Akt seinerseits inhibiert die Apoptose und stimuliert die Progression des Zellzyklus sowie die Zellproliferation [36]. Die Expression von phosphoryliertem Akt nimmt mit der Melanomprogression zu und korreliert invers mit dem Patientenüberleben $[13,44]$. Ein Heterozygotieverlust des PTEN-Locus auf Chromosom 10q wurde in etwa $30-50 \%$ [25, 40] und Mutationen von PTEN in 10\% aller Melanome gefunden [23]. Eine selektive Aktivierung von Akt konnte bei bis zu 60\% nichtfamiliärer Melanomen nachgewiesen werden [44]. Die Deregulation von Akt umfasst einerseits eine Überexpression infolge der Erhöhung der Anzahl von Genkopien und andererseits eine verminderte Funtion des PTEN-Proteins durch Verlust des PTEN-Gens [44].

Mutationen im N-RAS-Gen und genetische Alterationen in PTEN scheinen sich zumindest in Melanomzelllinien zu ergänzen. Zelllinien mit einer Mutation in dem einen Gen haben nur ausnahmsweise eine Mutation im anderen Gen [47]. RAS kann die $\mathrm{PI}_{3} \mathrm{~K}$ stimulieren, die ihrerseits die Konzentration von Phosphatidylinositol-3-Triphosphaten erhöht [15]. Mutiertes
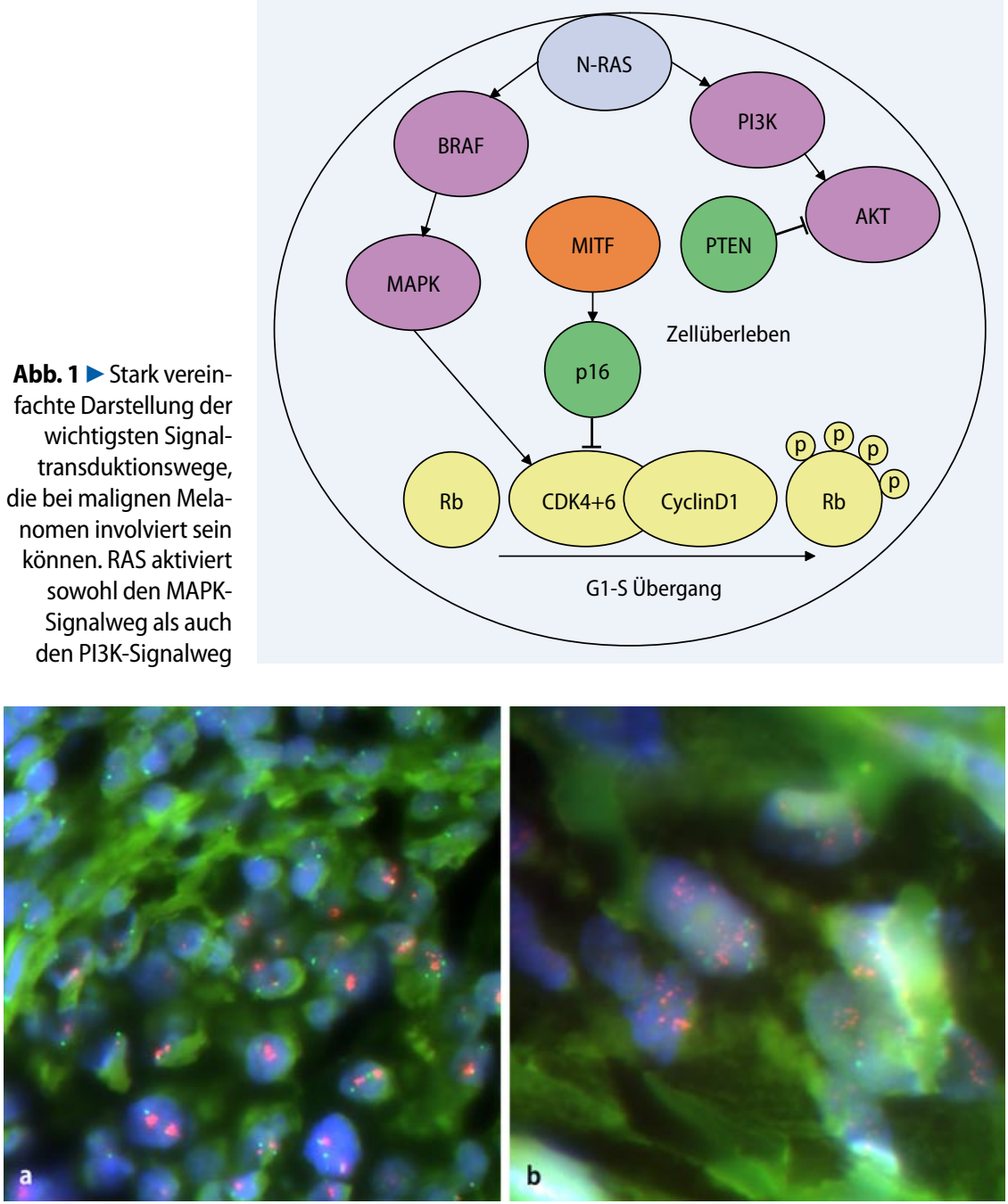

Abb. $2 \Delta$ a CyclinD1-Amplifikation in einem Melanom von chronisch sonnenbelasteter Haut. Die großen Tumorzellen in der rechten unteren Bildhälfte enthalten teils gruppierte vermehrte rote Gensignale und eine normale Anzahl grüne Zentromersignale. b Amplifikation des MDM 2-Gens in einem akralen Melanom. Die roten Gensignale sind deutlich vermehrt

PTEN kann diese nicht dephosphorylieren und wirkt somit synergistisch zu aktiviertem $N$-RAS.

\section{Genetische Veränderungen und anatomische Lokalisation}

Maligne Melanome lassen sich aufgrund ihrer anatomischen Lokalisation und der damit verbundenen Sonnenexposition gruppieren in Melanome von chronisch oder intermittierend sonnenexponierter Haut, akrale Melanome, Mukosamelanome und Uveamelanome. Am häufigsten entwickeln sich Melanome in intermittierend sonnenexponierter Haut, bei Männern am Rücken und bei Frauen an den Beinen. Menschen, die im Freien arbeiten und chronisch sonnenexponiert sind, entwickeln dagegen seltener Melanome [20]. Für die Entstehung von Melanomen in den verschiedenen Lokalisationen wurden unterschiedliche Mechanismen identifiziert ( $\bullet$ Tab. 1). Whiteman et al. [52, 53] wiesen nach, dass p53-positive Melanome bevorzugt im Kopf-HalsBereich und an den unteren Extremitäten vorkommen und assoziiert sind mit der Unfähigkeit zur Hautbräunung sowie einer Anamnese von nichtmelanozytären Hauttumoren. p53-negative Tumoren waren dagegen mit der Nävusdichte und mit Sommersprossen assoziiert.

$B R A F$-Mutationsanalysen ergaben unterschiedliche Mutationshäufigkeiten in Abhängigkeit der anatomischen Lokalisation des Primärtumors. Mutationen fanden sich bei bis zu 59\% der Tumoren von 
Tab. 1 Genetische Veränderungen von Melanomen in Abhängigkeit der anatomischen

Lokalisation des Primärtumors

\begin{tabular}{llll} 
Lokalisation & $\begin{array}{l}\text { BRAF/N-RAS- } \\
\text { Mutation }\end{array}$ & $\begin{array}{l}\text { Mutation und/oder } \\
\text { multiple Kopien } \\
\text { von KIT }\end{array}$ & $\begin{array}{l}\text { Andere häufige genetische } \\
\text { Veränderungen }\end{array}$ \\
$\begin{array}{l}\text { Haut mit intermittie- } \\
\text { render UV-Belastung }\end{array}$ & Häufig $[12,33]$ & Nein [11] & Verlust von Chromosom 10 [12] \\
$\begin{array}{l}\text { Haut mit chronischer } \\
\text { UV-Belastung }\end{array}$ & Selten $[12,33]$ & Ja [11] & $\begin{array}{l}\text { Erhöhte Kopienzahl des } \\
\text { CyclinD1-Gens [12, 22] }\end{array}$ \\
Akrale Haut & Selten $[12,29]$ & Ja [11] & $\begin{array}{l}\text { Amplifikationen in frühen } \\
\text { Tumorstadien [1, 2] }\end{array}$ \\
Mukosa & Selten $[12,18]$ & Ja [11] & $\begin{array}{l}\text { Amplifikationen [12] } \\
\text { Uvea }\end{array}$ \\
Selten $[17,56]$ & Nein [37] & $\begin{array}{l}\text { Chromosomenverluste }(1 p, 3, \\
6 q) \text { und -zugewinne (6p, 8q) } \\
{[22,28]}\end{array}$ \\
\hline & & &
\end{tabular}

intermittierend sonnenexponierter Haut und sind dort signifikant häufiger als in den übrigen Lokalisationen [12, 33]. Melanome von chronisch sonnenexponierter Haut zeigen dagegen in der komparativen genomischen Hybridisierung (CGH) signifikant mehr Amplifikationen als solche von intermittierend sonnenexponierter Haut ([2]; • Abb. 2 a).

Akrale, mukosale und uveale Melanome weisen nur sehr selten $B R A F-\mathrm{Mu}-$ tationen auf $[17,18,33,46]$. Akrale und mukosale Melanome sind charakterisiert durch häufigere chromosomale Aberrationen und Genamplifikationen als die übrigen Melanome, wobei in jeder Lokalisation distinkte Regionen des Genoms betroffen sind [2, 12, 49]. Die Amplifikationen beim akralen Melanom (• Abb. 2 b) sind im Gegensatz zu vielen anderen Tumoren ein frühes Ereignis und schon in nichtinvasiven In-situ-Läsionen vorhanden $[1,24]$. Amplifikationen konnten auch in bis zu $3 \mathrm{~mm}$ vom Primärtumor entfernten und zytologisch unauffälligen isolierten Melanozyten nachgewiesen werden [1]. Dies könnte eine Erklärung für die hohe Rate an Lokalrezidiven bei akralen Tumoren sein [48].

Curtin et al. [11] konnten zeigen, dass Melanome von aktinisch geschädigter Haut, akrale und mukosale Melanome gehäuft Mutationen oder erhöhte Kopienzahlen des KIT-Gens aufweisen, während Veränderungen des KIT-Gens in Melanomen von intermittierend UV-exponierter Haut in keinem von 18 Fällen nachweisbar waren. Die Autoren weisen darauf hin, dass das häufig beobachtete lentiginöse Wachstumsmuster der von Verände- rungen des Gens betroffenen Melanomsubtypen möglicherweise mit der Funktion von KIT bei der Migration und dem „Homing“ der Melanozyten in der basalen Epidermis zusammenhängen könnte.

Uveamelanome sind gekennzeichnet durch typische Chromosomenverluste (1p, 3, 6q) und -zugewinne (6p, 8q; [28]). Die häufige Kombination einer Monosomie des Chromosoms 3 mit zusätzlichen Kopien von $8 \mathrm{q}$ ist mit einer schlechten Prognose assoziiert [43]. Mutationen von KIT konnten wir in einer eigenen Untersuchung an 101 Uveamelanomen nicht nachweisen [37].

\section{Bedeutung für die Diagnostik}

Von der molekularpathologischen Diagnostik erhoffen wir uns eine Hilfestellung bei der Ermittlung der Diagnose, Subklassifizierung, Prognose, Therapieauswahl und Therapieantwort von Tumoren. Molekularpathologische Untersuchungen haben jedoch noch keinen Eingang in die Routinediagnostik des malignen Melanoms gefunden. Dieser Umstand wird erklärt durch das Fehlen von einfach und kostengünstig analysierbaren molekularen Markern, die im Einzelfall eine klare Aussage bezüglich Dignität oder Prognose der melanozytären Läsion erlauben. Sehr oft zeigen diese Marker kontinuierliche Veränderungen von gewöhnlichen Nävi zu dysplastischen Nävi zu Melanomen in radiärer bzw. vertikaler Wachstumsphase und schließlich zum metastasierenden Melanom [8].

Einige Zusatzuntersuchungen wie beispielsweise die immunhistochemische
Expression von Ki-67 und anderen Zellzyklusproteinen [8], BRAF-Mutationsanalysen [38], CGH $[2,3,12]$ oder Genexpressionsarrays [6] erhöhen die Sensitivität und Spezifität der Diagnose von histologisch unklaren melanozytären Läsionen. Da das Resultat der Zusatzuntersuchungen aufgrund der biologischen Heterogenität melanozytärer Läsionen nie in allen benignen bzw. malignen Tumoren einheitlich ausfällt, ist die Aussagekraft im Einzelfall aber beschränkt und muss durch den erfahrenen Pathologen in $\mathrm{Zu}$ sammenschau mit den übrigen morphologischen und klinischen Befunden beurteilt werden.

Die vermehrte Berücksichtigung der anatomischen Lokalisation und die Quantifizierung der solaren Elastose in der Tumorumgebung als approximatives $\mathrm{Ma} \beta$ für die Sonnenexposition könnten für das Studium genetischer Unterschiede im Rahmen von Forschungsprojekten relevant sein.

Die Analyse bestimmter molekularer Veränderungen zur Selektion von Patienten für eine gezielte molekulare Therapie ist zum jetzigen Zeitpunkt Zentren vorbehalten, an denen entsprechende Therapiestudien laufen [4]. Anhand der Entwicklung der molekularen Diagnostik bei anderen Tumortypen wie dem Mammakarzinom oder dem Kolonkarzinom ist aber vorauszusehen, dass solche Aufgaben in den nächsten Jahren auch auf die Dermatopathologen zukommen werden [8].

\section{Bedeutung für die Therapie}

Das Überleben von Melanompatienten mit inoperablen Fernmetastasen lässt sich nach wie vor therapeutisch kaum verbessern [19]. Große Hoffnungen werden deshalb in neue Therapien gesetzt, die molekulare Ziele eines individuellen Tumors anvisieren.

Die vielversprechendsten Resultate wurden mit dem RAF-Inhibitor Sorafenib in Kombination mit Chemotherapie erzielt. Sorafenib blockiert die Aktivität von mutiertem und Wildtyp BRAF in Tumorzellen, wirkt aber auch gegen verschiedene Rezeptortyrosinkinasen, die bei der Neovaskularisation und Tumorprogression involviert sind [55]. Allerdings wurden bislang nur kleine bis mittelgroße Pha- 
se II klinische Studien beim metastasierten Melanom durchgeführt [4]. In Melanomen akraler, mukosaler und chronisch UV-exponierter Haut wurden KIT-Mutationen nachgewiesen, die auch bei Tumoren vorkommen, welche auf eine Imatinib-Therapie ansprechen [11]. Dieser Umstand lässt hoffen, dass der Subgruppe von Melanompatienten mit Mutation des KITGens mit einer Imatinib-Therapie geholfen werden könnte.

Daten aus Studien mit molekularen gezielten Therapien haben gezeigt, dass die Anwesenheit eines molekularen Ziels den therapeutischen Erfolg nicht automatisch garantiert [5]. Aufgabe der Forschung wird es einerseits sein, diejenigen molekularen Veränderungen zu identifizieren, die durch eine spezifische Therapie beeinflussbar sind. Andererseits müssen reproduzierbare und sensitive Marker für das Therapieansprechen etabliert werden.

Die Pharmaindustrie investiert immense Geldbeträge in die Entwicklung neuer Therapeutika. Forschungsgelder für die Ermittlung relevanter diagnostischer Parameter für die korrekte Selektion von Patientensubgruppen durch den Pathologen, die auf eine bestimmte Therapie eine hohe Ansprechwahrscheinlichkeit haben werden, fließen dagegen deutlich spärlicher. Wenn die neuen gezielten Therapien effektiv und bezahlbar sein sollen, muss dieses Missverhältnis in der Forschungsförderung reduziert werden.

\section{Ausblick}

Die konventionelle Lichtmikroskopie wird auch in Zukunft einen zentralen Stellenwert in der Melanomdiagnostik einnehmen. In einzelnen Fällen werden als Ergänzung molekulare Zusatzuntersuchungen zur Anwendung gelangen, die in Studien mit eindeutigen Melanomfällen etabliert wurden. Aktuelle Forschungsanstrengungen zielen durch die Kombination mehrerer molekularer Marker auf eine Verbesserung der Voraussagekraft des Metastasierungsrisikos eines individuellen Tumors im Vergleich zum herkömmlichen klinisch-pathologischen Staging [8]. Viele dieser Marker könnten auch potenzielle Therapieziele abgeben, die jedoch aufgrund der genetischen Heterogenität melanozytärer Tu-

Pathologe 2007 · 28:474-478 DOI 10.1007/s00292-007-0942-6

(c) Springer Medizin Verlag 2007

K. Glatz

Molekulare Heterogenität maligner Melanome

\section{Zusammenfassung}

Maligne Melanome bilden eine heterogene Gruppe von Tumoren, die in Abhängigkeit von ihrer anatomischen Lokalisation und der UV-Exposition charakteristische genetische Aberrationen aufweisen. Eine Aktivierung des Mitogen-aktivierte-Proteinkinase- (MAPK)Signaltransduktionsweges findet sich bei einem Großteil aller Melanome, wobei entweder eine somatische Missense-Mutation von BRAF oder deutlich seltener eine Mutation von $N$-RAS vorliegt. Der Verlust der beiden Produkte des CDKN2A-Gens, p16 ${ }^{\mathrm{ARF}}$ und p14 ${ }^{\text {INK4a }}$, oder die Amplifikation von Microphthalmia-assoziiertem Transkriptionsfaktor (MITF) prädisponieren ebenfalls zur Melanomentwicklung. BRAF-Mutationen werden vor allem in Melanomen von intermittierend UV-exponierter Haut beobachtet. Akrale und mukosale Melanome sowie solche von chronisch UV-geschädigter Haut sind durch dis-

tinkte Muster chromosomaler Aberrationen mit häufigen Amplifikationen sowie Alterationen des KIT-Gens gekennzeichnet, während BRAF-Mutationen in diesen Lokalisationen selten nachweisbar sind. Uveamelanome zeigen wiederkehrende Chromosomenverluste $(1 p, 3,6 q)$ und Zugewinne (6p, 8q), aber kaum BRAF-Mutationen. Bis heute haben molekulare Untersuchungen noch keinen festen Platz in der Routinediagnostik des malignen Melanoms. Die Entwicklung gezielter molekularer Therapeutika wird es aber zukünftig notwendig machen, mittels molekularpathologischer Methoden diejenigen Melanompatienten zu identifizieren, die von einer bestimmten Therapie am ehesten profitieren.

Schlüsselwörter

Melanom · Genetische Veränderungen . Mutationen · MAPK-Signalweg · Transkriptionsfaktoren

\section{Molecular heterogeneity of malignant melanomas}

\section{Abstract}

Malignant melanomas make up a heterogeneous group of tumors characterized by particular genetic aberrations depending on their anatomic localization and UV exposure. Activation of the mitogen-activated protein kinase (MAPK) signaling pathway is found in the majority of melanomas, with either somatic missense mutations of BRAF or, considerably more rarely, mutations of $N-R A S$. The loss of both products of the CDKN2A gene, proteins $\mathrm{p} 16^{\mathrm{ARF}}$ and $\mathrm{p} 14^{\mathrm{INK} 4 \mathrm{a}}$, or amplification of microphthalmia-associated transcriptional factor (MITF) are also predisposing factors in the development of melanoma. BRAF mutations are observed mainly in melanomas on skin liable to intermittent UV exposure. Acral and mucosal melanomas, and also melanomas on skin damaged by chronic exposure to the sun are characterized by distinct patterns of chromosomal aberrations with frequent amplifications and alterations of the KIT gene, while $B R A F$ mutations are rarely found in these sites. Uveal melanomas show recurrent chromosomal losses (1p, 3, 6q) and gains $(6 p, 8 q)$, but mutations of BRAF are hardly ever found. So far, ancillary molecular studies are not regularly applied in the routine diagnostic procedures performed when malignant melanoma is suspected. In the future, however, the development of targeted molecular therapies will require that molecular pathological techniques are used to identify the melanoma patients who will most probably benefit from a particular therapy.

\section{Keywords}

Melanoma - Genetic alterations · Mutations . MAPK signaling pathway - Transcriptional factors 
moren nur bei einer Subgruppe wirksam sein dürften. Entsprechend wird es in $\mathrm{Zu}$ kunft unsere Aufgabe sein, für eine Therapie infrage kommende Patienten mittels molekulardiagnostischer Tests zu identifizieren.

\section{Fazit für die Praxis}

Verschiedene Wege können zum Melanom führen. Diese sind oftmals für eine bestimmte anatomische Lokalisation typisch. Bei der Erforschung genetischer Veränderungen melanozytärer Tumoren sollte deshalb der Tumorlokalisation vermehrt Beachtung geschenkt werden. Trotz zahlreicher neuer Erkenntnisse zur Genetik des malignen Melanoms, stellt die konventionell histologische Beurteilung melanozytärer Tumoren nach wie vor den diagnostischen Goldstandard dar. Die genetischen Heterogenität maligner Melanome und die Komplexität der involvierten Mechanismen erschwert die Entwicklung effektiver gezielter Therapeutika für fortgeschrittene Tumoren. Aufgabe der Pathologen wird es sein, die für eine solche gezielte Therapie infrage kommenden Melanompatienten zu identifizieren.

\section{Korrespondenzadresse \\ PD Dr. K. Glatz \\ Institut für Pathologie \\ Schönbeinstraße 40, 4031 Basel, Schweiz \\ Katharina.Glatz@unibas.ch}

Interessenkonflikt. Der korrespondierende Autor gibt an, dass kein Interessenkonflikt besteht.

\section{Literatur (Auswahl)}

1. Bastian BC, Kashani-Sabet M, Hamm H et al. (2000) Gene amplifications characterize acral melanoma and permit the detection of occult tumor cells in the surrounding skin. Cancer Res 60: 1968-1973

2. Bastian BC, Olshen AB, LeBoit PE, Pinkel D (2003) Classifying melanocytic tumors based on DNA copy number changes. Am J Pathol 163: 1765-1770

6. Bittner M, Meltzer P, Chen Y et al. (2000) Molecular classification of cutaneous malignant melanoma by gene expression profiling. Nature 406: 536-540

8. Carlson JA, Ross JS, Slominski A et al. (2005) Molecular diagnostics in melanoma. J Am Acad Dermatol 52: 743-775; quiz 775-748

10. Cree IA (2000) Cell cycle and melanoma-two different tumours from the same cell type. J Pathol 191 112-114

12. Curtin JA, Fridlyand J, Kageshita T et al. (2005) Distinct sets of genetic alterations in melanoma. $\mathrm{N}$ Engl J Med 353: 2135-2147
13. Dai DL, Martinka M, Li G (2005) Prognostic significance of activated Akt expression in melanoma: a clinicopathologic study of 292 cases. J Clin Oncol 23: 1473-1482

14. Davies H, Bignell GR, Cox C et al. (2002) Mutations of the BRAF gene in human cancer. Nature 417: 949-954

15. Downward J (1997) Role of phosphoinositide-3$\mathrm{OH}$ kinase in Ras signaling. Adv Second Messenger Phosphoprotein Res 31: 1-10

17. Edmunds SC, Cree IA, Di Nicolantonio F et al. (2003) Absence of BRAF gene mutations in uveal melanomas in contrast to cutaneous melanomas. Br J Cancer 88: 1403-1405

18. Edwards RH, Ward MR, Wu H et al. (2004) Absence of BRAF mutations in UV-protected mucosal melanomas. J Med Genet 41: 270-272

20. Elwood JM, Jopson J (1997) Melanoma and sun exposure: an overview of published studies. Int J Cancer 73: 198-203

21. Garraway LA, Widlund HR, Rubin MA et al. (2005) Integrative genomic analyses identify MITF as a lineage survival oncogene amplified in malignant melanoma. Nature 436: 117-122

22. Glatz-Krieger K, Pache M, Tapia C et al. (2006) Anatomic site-specific patterns of gene copy number gains in skin, mucosal, and uveal melanomas detected by fluorescence in situ hybridization. Virchows Arch 449: 328-333

25. Isshiki K, Elder DE, Guerry D, Linnenbach AJ (1993) Chromosome 10 allelic loss in malignant melanoma. Genes Chromosomes Cancer 8: 178-184

26. Jhappan C, Noonan FP, Merlino G (2003) Ultraviolet radiation and cutaneous malignant melanoma. Oncogene 22: 3099-3112

28. Kilic E, Gils W van, Lodder E et al. (2006) Clinical and cytogenetic analyses in uveal melanoma. Invest Ophthalmol Vis Sci 47: 3703-3707

29. Lang J, MacKie RM (2005) Prevalence of exon 15 BRAF mutations in primary melanoma of the superficial spreading, nodular, acral, and lentigo maligna subtypes. J Invest Dermatol 125: 575-579

32. Loercher AE, Tank EM, Delston RB, Harbour JW (2005) MITF links differentiation with cell cycle arrest in melanocytes by transcriptional activation of INK4A. J Cell Biol 168: 35-40

33. Maldonado JL, Fridlyand J, Patel H et al. (2003) Determinants of BRAF mutations in primary melanomas. J Natl Cancer Inst 95: 1878-1890

34. Michaloglou C, Vredeveld LC, Soengas MS et al. (2005) BRAFE600-associated senescence-like cell cycle arrest of human naevi. Nature 436: 720-724

35. Miller AJ, Mihm MC Jr (2006) Melanoma. N Engl J Med 355: 51-65

37. Pache M, Glatz K, Bosch D et al. (2003) Sequence analysis and high-throughput immunhistochemical profiling of KIT (CD 117) expression in uveal melanoma using tissue microarrays. Virchows Arch 443: 741-744

39. Pollock PM, Harper UL, Hansen KS et al. (2003) High frequency of BRAF mutations in nevi. Nat Genet 33: 19-20

41. Satyamoorthy K, Herlyn M (2003) p16INK4A and familial melanoma. Methods Mol Biol 222: 185195

47. Tsao H, Zhang X, Fowlkes K, Haluska FG (2000) Relative reciprocity of NRAS and PTEN/MMAC1 alterations in cutaneous melanoma cell lines. Cancer Res 60: 1800-18046

53. Whiteman DC, Watt P, Purdie DM et al. (2003) Melanocytic nevi, solar keratoses, and divergent pathways to cutaneous melanoma. J Natl Cancer Inst 95: 806-812
54. Whiteman DC, Whiteman CA, Green AC (2001) Childhood sun exposure as a risk factor for melanoma: a systematic review of epidemiologic studies. Cancer Causes Control 12: 69-82

55. Wilhelm SM, Carter C, Tang L et al. (2004) BAY 439006 exhibits broad spectrum oral antitumor activity and targets the RAF/MEK/ERK pathway and receptor tyrosine kinases involved in tumor progression and angiogenesis. Cancer Res 64: 7099-7109

56. Zuidervaart W, Nieuwpoort F van, Stark M et al. (2005) Activation of the MAPK pathway is a common event in uveal melanomas although it rarely occurs through mutation of BRAF or RAS. Br J Cancer 92: 2032-2038

Das komplette Literaturverzeichnis ...

... finden Sie in der elektronischen Version dieses Beitrags unter www.DerPathologe.de 